may be due to abnormalities of a single or many pulmonary arteries. Clearly it is vitally important to make an adequate diagnosis of the problem before taking a biopsy sample which can have fatal results as demonstrated in the second case. Most hospitals will have available adequate imaging technology. If there is any doubt at all the patient should be referred for spiral CT scanning which can successfully demonstrate clot or other abnormalities in the pulmonary artery and then, if the doubt persists, proceed to angiography. Only when the diagnosis is really clear should a biopsy sample be taken. Many would argue that this should only be done where adequate surgical control of any subsequent bleeding is possible. In most cases local pulmonary artery disease occurs in young, otherwise fit, people who have an excellent prognosis provided a firm diagnosis can be reached before any biopsy procedure.

\section{Chronic lung abscess due to Pasteurella multocida}

Philippe Machiels, Jean-Paul Haxhe, Jean-Paul Trigaux, Monique Delos, Jean-Claude Shoevaerdts, Olivier Vandenplas

\begin{abstract}
Medicine
P Machiels

$O$ Vandenplas

\section{Department of Thoracic Surgery J-P Haxhe}

J-C Shoevaerdts

\section{Department of Radiology J-P Trigaux}

Department of Chest

\section{Department of Pathology M Delos}

University Hospital of Mont-Godinne, 5530-Yvoir, Belgium

Reprint requests to: Dr O Vandenplas.

Received 1 July 1994 Returned to authors 15 September 1994 Revised version received 11 October 1994 Accepted for publication 19 October 1994

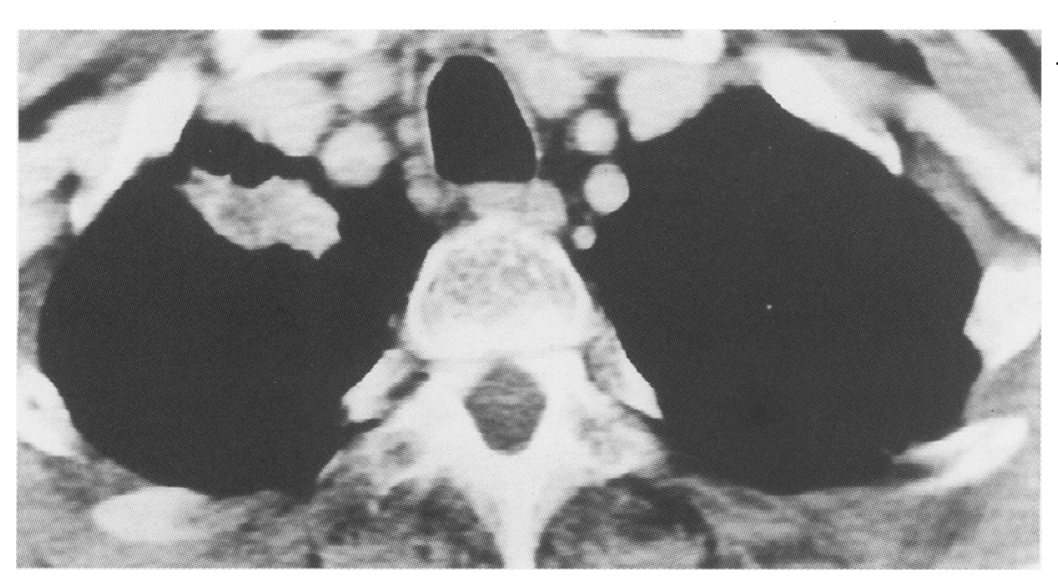

Computed tomographic scan of the chest showing a heterogeneous lesion with spiculated

borders in the apical segment of the right upper lobe.

\begin{abstract}
A case of chronic lung abscess due to Pasteurella multocida presenting as a solitary pulmonary mass with a computed tomographic appearance suggestive of malignancy is described.

(Thorax 1995;50:1017-1018)
\end{abstract}

Keywords: lung abscess, Pasteurella.

Solitary pulmonary masses frequently remain a difficult diagnostic challenge even though computed tomographic (CT) scanning and percutaneous needle biopsy have greatly improved the distinction between benign and malignant lesions. We present a case of a chronic lung abscess due to Pasteurella multocida which simulated a malignant process.

\section{Case report}

A 70 year old ex-smoker presented with purulent sputum and haemoptysis 10 years after left lower lobectomy for a T2NOMO adenocarcinoma. He experienced mild chronic bronchitis but continued to work as a farmer. The chest radiograph showed a small ill defined opacity in the right retroclavicular area. Sputum culture grew Pasteurella multocida and Moraxella catarrhalis. The patient was treated with doxycycline $100 \mathrm{mg}$ daily for two weeks. Although the symptoms subsided, the chest radiograph showed persistence of the opacity. Physical examination was non-contributory. Erythrocyte sedimentation rate was $37 \mathrm{~mm}$ in the first hour and the white blood cell count was normal. Spirometric measurements showed a forced expiratory volume in one second $\left(\mathrm{FEV}_{1}\right)$ of 2.051 (67\% predicted) and a forced vital capacity (FVC) of 4.751 (120\% predicted). The $\mathrm{FEV}_{1}$ improved by $22 \%$ after inhaled bronchodilator. A chest CT scan revealed a $2 \mathrm{~cm}$ lesion with a central area of low attenuation and spiculated borders in the apical segment of the right upper lobe. Bronchoscopic examination showed patent bronchi but brush cytological specimens from the right upper lobe yielded atypical cells. Bacteriological studies of bronchial lavage fluid, including cultures for mycobacteria and fungi, remained negative. Percutaneous needle biopsy under CT guidance resulted in parenchymal haemorrhage and haemoptysis which resolved spontaneously after 24 hours. Cytological examination showed non-specific inflammatory cells. No material could be collected for bacteriological studies.

The lesion remained unchanged on a CT scan performed three months later, but appeared to have enlarged by about $50 \%$ by six months (figure). Another bronchoscopic examination with cytological and bacteriological studies of bronchial lavage fluid and brushings remained negative. Because of the increasing suspicion of malignancy, the patient underwent resection of the right upper lobe. Histological examination showed an accumulation of neutrophils surrounded by fibrosis without evidence of malignancy. Culture of the surgical specimen grew $P$ multocida sensitive to penicillins and tetracyclines. On questioning, the patient denied any animal bite, but had been exposed to dogs and cattle. He was treated with amoxycillin/clavulanic acid $4 \mathrm{~g}$ 
intravenously daily for two weeks and then $2 \mathrm{~g}$ orally for four weeks. At follow up 18 months after lobectomy he remained well.

\section{Discussion}

Pasteurella multocida is a Gram negative coccobacillus which is frequently recovered from the nasopharynx or gastrointestinal tract of domestic and wild mammals (cats, dogs, cattle, swine, sheep, and rats) and birds (chickens, turkeys, and ducks). ${ }^{1}$ The organism causes epidemic fowl cholera in birds and haemorrhagic septicaemia in mammals. In humans $P$ mul tocida is responsible for soft tissue infections after animal bites or scratches which are occasionally associated with arthritis or osteomyelitis. $P$ multocida infection has also been reported after non-traumatic animal exposure and $5-15 \%$ of affected subjects have no known exposure to animals. Various systemic infections including septicaemia, meningitis, and peritonitis have been described. Lower respiratory tract infections including pneumonia, empyema, and lung abscess have been documented in subjects with underlying chronic pulmonary disease ${ }^{23}$ and, more recently, in one subject with AIDS. ${ }^{4}$

Our patient presented with a pulmonary lesion which had features on the CT scan consistent with a malignant process. Isolation of pure $P$ multocida from lung specimens obtained at surgery provided definitive evidence that this organism was responsible for a chronic abscess. To our knowledge, only three cases of lung abscess due to $P$ multocida have been reported in the literature. ${ }^{5-7}$ Two of these cases involved acutely ill patients in whom the disease was complicated by empyema or massive haemoptysis. ${ }^{56}$ In contrast, Steyer et al described a case of lung abscess due to $P$ multocida in an asymptomatic patient with fibrotic changes due to old tuberculosis. Although the course of the disease in our patient might have been influenced by the initial treatment with doxycycline, our observation confirms that $P$ multocida has the potential to cause a chronic lung abscess with an insidious clinical presentation. Penicillin derivatives and tetracyclines are considered the drugs of choice for treatment of $P$ multocida infections. ${ }^{1}$ This observation suggests that pulmonary infections require prolonged antibiotic therapy in order to prevent a chronic course, although the optimum dosage and duration of treatment remain uncertain.

Despite its apparent rarity, chronic abscess due to $P$ multocida should be considered in the differential diagnosis of solitary pulmonary masses, especially in subjects exposed to domestic pets or cattle. The presence of $P$ multocida in sputum culture should not be neglected even though the organism has occasionally been isolated from the nasopharynx of healthy subjects exposed to animals. ${ }^{1} \mathrm{Ma}$ terial from percutaneous needle aspiration biopsies should be systematically obtained for bacteriological analysis.

1 Weber DJ, Wolfson JS, Swartz MN, Hooper DC. Pasteurella multocida infections, Swartz MN, Hooper DC. Pasteurella multocida infections. Report of 34 cases

2 Nelson SC, Hammer GS. Pasteurella multocida empyema: case report and review of the literature. $A m \mathcal{F}$ Med $S$ 1981;281:43-9.

3 Rose HD, Mathai G. Acute Pasteurella multocida pneumonia. Br $\mathcal{F}$ Dis Chest 1977;71:123-6.

4 Drabick JJ, Gasser RA, Saunders NB, Hadfield TL, Rogers LC, Berg BW, et al. Pasteurella multocida pneumonia in a man with AIDS and nontraumatic feline exposure. Chest man with AlDS

5 Maneche HC, Toll HW. Pulmonary cavitation and massive hemorrhage caused by Pasteurella multocida. Report of a hemorrhage caused by Pasteurella mu

6 Schmidt EC, Truitt LV, Koch ML. Pulmonary abscess with empyema caused by Pasteurella multocida. Am $\mathcal{J}$ Clin Patho 1970;54:733-6.

7 Steyer J, Sobonya RE. Pasteurella multocida lung abscess. Case report and review of the literature. Arch Intern Med 1984, 144:1081-2 